\title{
WARM SRAM: A Novel Scheme to Reduce Static Leakage Energy in SRAM Arrays
}

\author{
Mahadevan Gomathisankaran Akhilesh Tyagi \\ lowa State University lowa State University \\ gmdev@iastate.edu tyagi@iastate.edu
}

(1) Introduction

(2) Proposed Circuit Technique

(3) Reducing static energy in On-Chip Caches

(4) Model Validity

(5) Conclusion and Future Work 


\section{INTRODUCTION}

Expected increase in the static leakage current

$\rightarrow$ Feature Size to reach $22 \mathrm{~nm}$ in 2016

$\rightarrow$ Leakage current to increase by factor of $1 \mathrm{~K}-10 \mathrm{~K}$ in going from $180 \mathrm{~nm}$ to $70 \mathrm{~nm}$

Leakage current will play a major role in circuit design

$\rightarrow$ Not only arrays but also high fan-out logic will be affected

New design methodologies have to be invented to avoid Red Brick Wall

$\rightarrow$ We propose warmup-CMOS which uses depletion mode transistors 


\section{SUBTHRESHOLD LEAKAGE IN CMOS}

Various leakage mechanisms

$\rightarrow$ PN Reverse Bias, Weak Inversion, DIBL, GIDL, Punchthrough

Leakage Current

$$
\begin{aligned}
& I_{\text {sub }}=A * \exp \left\langle\frac{q}{n^{\prime} k T}\left(V_{g}-V_{s}-V_{t h 0}-\gamma^{\prime} V_{s}+\eta V_{d s}\right)\right\rangle * B \\
& A=\mu_{0} C_{o x} \frac{W_{\text {eff }}}{L_{\text {eff }}}\left\langle\frac{k T}{q}\right\rangle^{2} e^{1.8} \\
& B=1-\exp \left(\frac{-q V_{d s}}{k T}\right)
\end{aligned}
$$




\section{SUBTHRESHOLD LEAKAGE IN CMOS}

Various leakage mechanisms

$\rightarrow$ PN Reverse Bias, Weak Inversion, DIBL, GIDL, Punchthrough

Leakage Current

$$
\begin{aligned}
& I_{\text {sub }}=A * \exp \left\langle\frac{q}{n^{\prime} k T}\left(V_{g}-V_{s}-V_{t h 0}-\gamma^{\prime} V_{s}+\eta V_{d s}\right)\right\rangle * B \\
& A=\mu_{0} C_{o x} \frac{W_{\text {eff }}}{L_{\text {eff }}}\left\langle\frac{k T}{q}\right\rangle^{2} e^{1.8} \\
& B=1-\exp \left(\frac{-q V_{d s}}{k T}\right)
\end{aligned}
$$




\section{EARLIER RESEARCH}

Gated- $\mathrm{V}_{d d}$

+ Interposes a high- $\mathrm{V}_{t}$ transistor between the circuit and one of the power supply rails

+ Reduces the leakage current of a normal transistor to effectively the leakage current of the high $-\mathrm{V}_{t}$ control transistor

- Contents of the cell are lost

- Control algorithm should be smart

ABB-MTCMOS

+ Dynamically raise $\mathrm{V}_{t}$ by modulating the back-gate bias voltage, i.e., $V_{t}$ $=V_{t 0}+\gamma\left(\sqrt{\phi_{b i}+V_{s b}}-\sqrt{\phi_{b i}}\right)$

- Higher energy/delay per transition and higher $\mathrm{V}_{d d+}$ offsets the leakage power savings 


\section{EARLIER RESEARCH}

Gated- $\mathrm{V}_{d d}$

+ Interposes a high- $\mathrm{V}_{t}$ transistor between the circuit and one of the power supply rails

+ Reduces the leakage current of a normal transistor to effectively the leakage current of the high $-\mathrm{V}_{t}$ control transistor

- Contents of the cell are lost

- Control algorithm should be smart

ABB-MTCMOS

+ Dynamically raise $\mathrm{V}_{t}$ by modulating the back-gate bias voltage, i.e., $V_{t}$ $=V_{t 0}+\gamma\left(\sqrt{\phi_{b i}+V_{s b}}-\sqrt{\phi_{b i}}\right)$

- Higher energy/delay per transition and higher $\mathrm{V}_{d d+}$ offsets the leakage power savings 


\section{DVS}

+ In sub-micron processes leakage current increases exponentially with supply voltage

+ Supply voltage is reduced to an optimum value (knee point of the curve, $1.5^{\star} \mathrm{V}_{t}$ )

+ Two-fold reduction (both voltage and current) of the leakage power is achieved

- Memory cell in standby (drowsy) mode cannot be read or written

What is Missing?

$\rightarrow$ A comprehensive solution which has low (much less) control overhead and still achieves the maximum possible leakage reduction

$\rightarrow$ Reduction is maximum if the circuit is in standby or low-leakage mode whenever it is not used 


\section{DVS}

+ In sub-micron processes leakage current increases exponentially with supply voltage

+ Supply voltage is reduced to an optimum value (knee point of the curve, $1.5^{\star} \mathrm{V}_{t}$ )

+ Two-fold reduction (both voltage and current) of the leakage power is achieved

- Memory cell in standby (drowsy) mode cannot be read or written

What is Missing?

$\rightarrow$ A comprehensive solution which has low (much less) control overhead and still achieves the maximum possible leakage reduction

$\rightarrow$ Reduction is maximum if the circuit is in standby or low-leakage mode whenever it is not used 


\section{Our Proposed Solution}

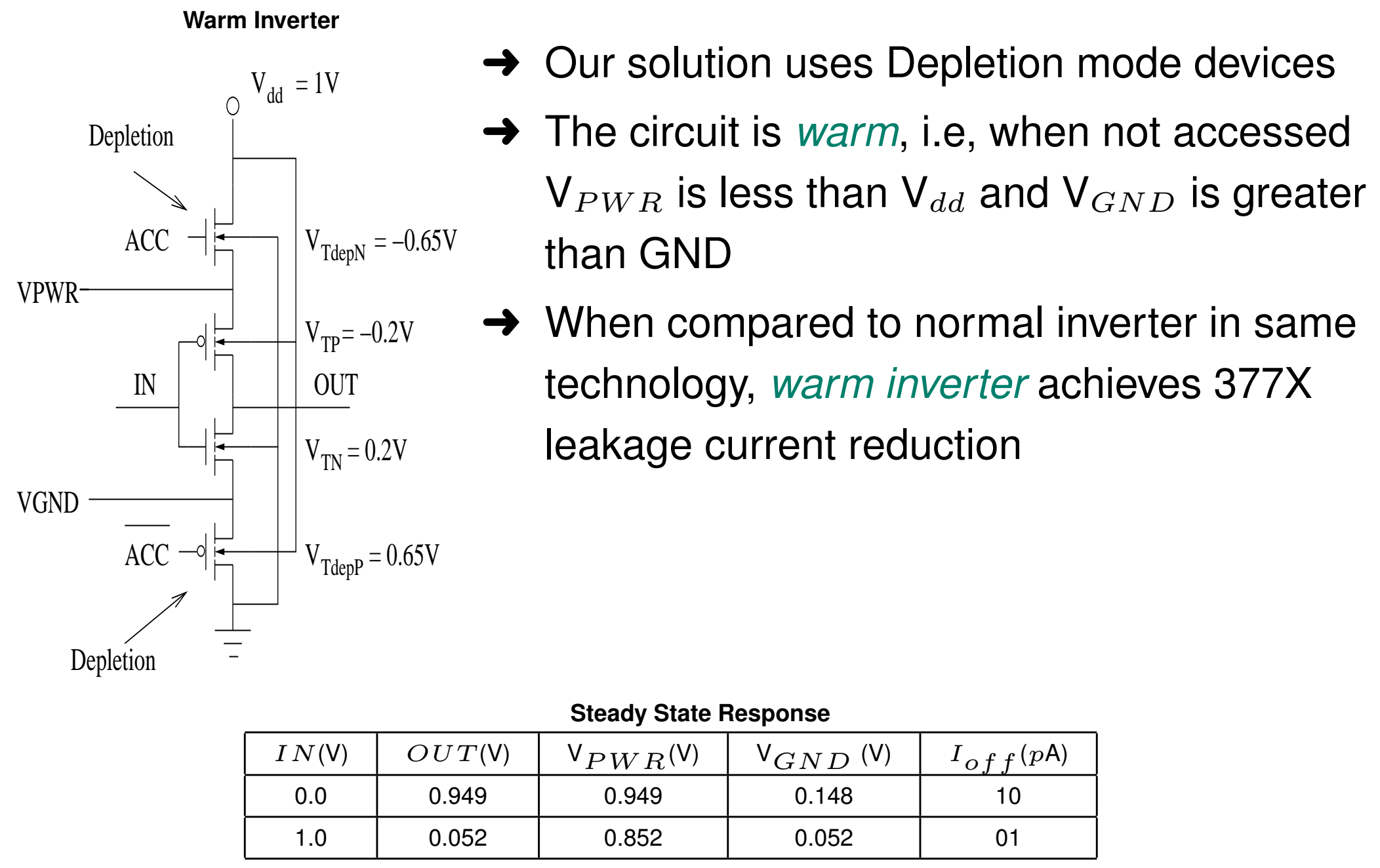




\section{Limitations:}

$\rightarrow$ Performance Penalty, as NMOS in the charging path and PMOS in the discharging path

$\rightarrow$ Energy Penalty, Extra Switching Energy $=\xi=0.3 * C_{\text {diff }} J$

$\rightarrow$ Cascading Effect, for a cross coupled inverter we get High $=742 \mathrm{mV}$, Low $=225 m V, \mathrm{I}_{\text {off }}=515 \mathrm{pA}$ (compare with actual $\left.\mathrm{I}_{\text {of } f} 6.25 \mathrm{AA}\right)$

\begin{tabular}{|c|c|c|c|c|}
\hline & ${ }^{t} p L H(p s)$ & $t_{p H L}(p s)$ & $t_{r}(p s)$ & $t_{f}(p s)$ \\
\hline Base & 16.8 & 10.54 & 33.63 & 17.31 \\
\hline New & 25.9 & 16.32 & 40.72 & 30.89 \\
\hline \%Inc & 54.2 & 54.80 & 21.10 & 78.50 \\
\hline
\end{tabular}




\section{ApplicATION TO CACHES}

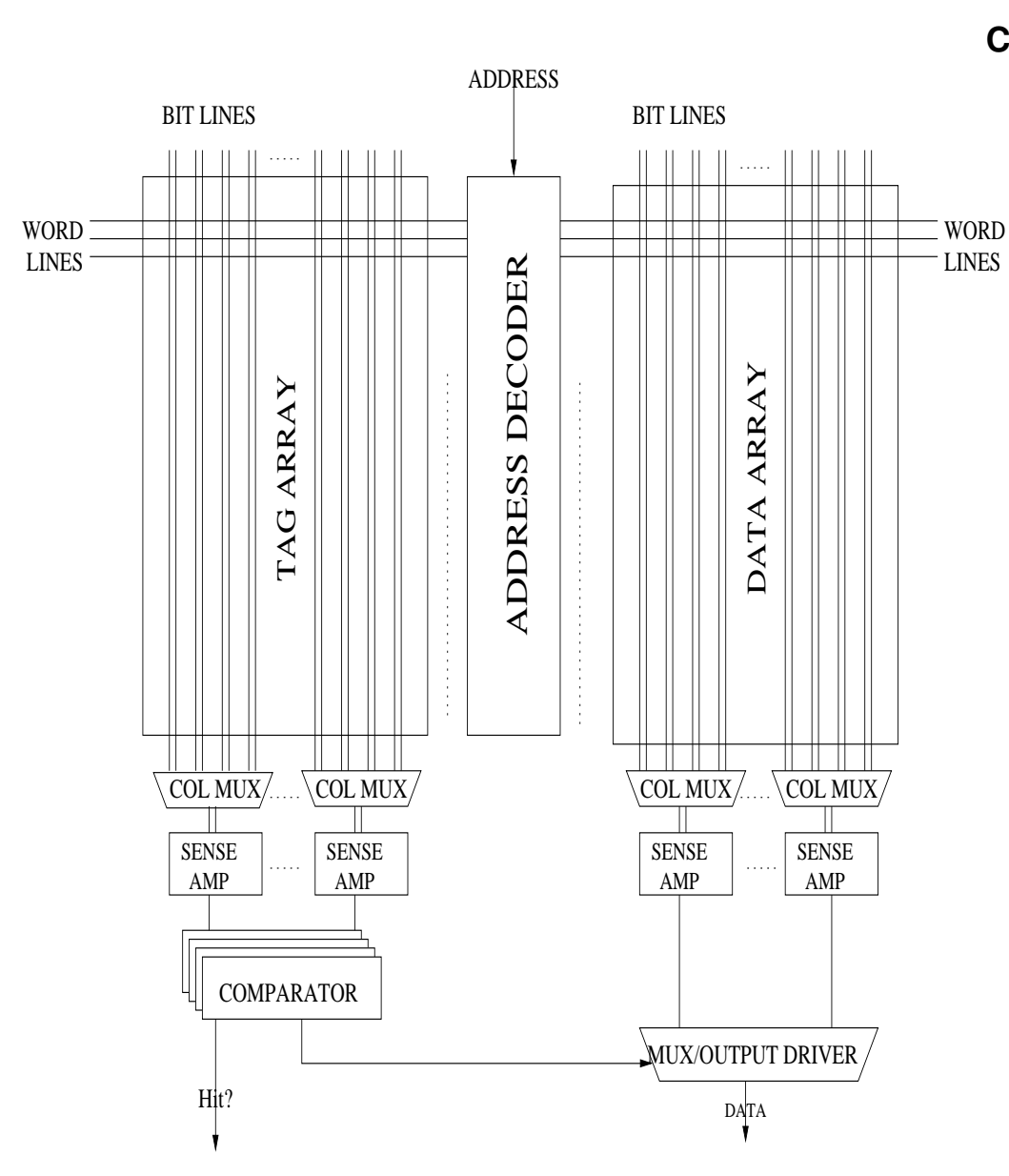

Cache Access Timing for a 32KB, 4-way, 1 RW Port, 1 Sub-bank Cache

\begin{tabular}{|c|c|c|}
\hline & Data Array Delay $(p s)$ & Tag Array Delay $(p s)$ \\
\hline Decoder & 208.572 & 099.410 \\
\hline Wordline & 115.975 & 044.415 \\
\hline Bitline & 011.765 & 011.898 \\
\hline Senseamp & 072.625 & 044.625 \\
\hline Compare & - & 112.912 \\
\hline Mux Driver & - & 150.077 \\
\hline Sel Inverter & - & 016.612 \\
\hline \hline Total & 408.936 & 479.949 \\
\hline
\end{tabular}

$\rightarrow \mathrm{L} 1$ cache sizes are typically 32KB - 64KB (Athlon has 128KB)

$\rightarrow \mathrm{L} 1$ miss rates are on the average $2 \%$

$\rightarrow$ On-Chip L2 caches are in the range of 256KB (Centrino has 1MB)

$\rightarrow$ We used CACTI 3.0 to find the cache access timing

Cache architecture of a $n$-way Set-Associative Cache 


\section{Simulation Setup:}

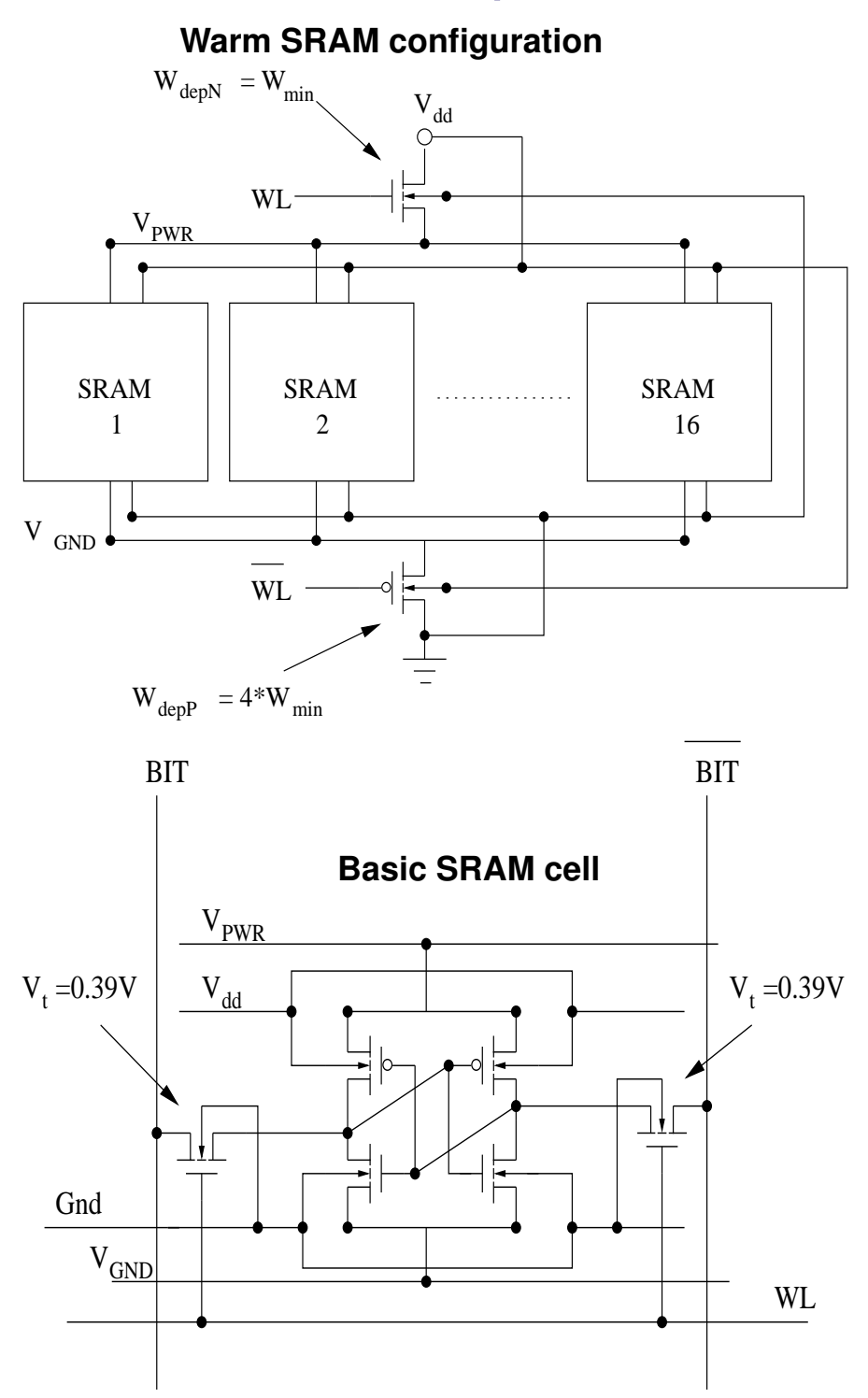

$\rightarrow$ A depletion device pair per cell would increase the area hence offset the energy savings

$\rightarrow$ The wordline access signal is used to control the depletion devices

$\rightarrow$ PMOS $_{d e p}$ is $4 \mathrm{~W}_{\text {min }}$, as cache read is in critical path this is justified

$\rightarrow$ Upto 6X increase in bitline delay (data array) will have no impact on cache access time

$\rightarrow$ Simulation is performed in HSPICE for a Subarray of size 128X256

$\rightarrow W L$ is not affected by addition of $16^{\star} \mathrm{C}_{g}$

$\rightarrow \overline{W L}$ is generated from $W L$ and since it is driving only $64^{*} \mathrm{C}_{g}$ it delay can be made one tenth of $W L$ 


\section{Leakage Reduction:}

$\rightarrow$ Leakage power reduction - 23X

$\rightarrow \mathrm{V}_{H}$ has moved closer to $\left|V_{T d e p N}\right|$, because one $\mathrm{NMOS}_{\text {dep }}$ is shared with 16 SRAM cells

$\rightarrow \mathrm{V}_{L}$ has moved closer to $V_{d d}-\left|V_{T d e p P}\right|$, but not as much as $\left|V_{H}\right|$, because width of $\mathrm{PMOS}_{d e p}$ has been increased

Steady State Response of a WARM SRAM Cell

\begin{tabular}{|c|c|c|}
\hline Param & Base & Warm SRAM \\
\hline$I_{L}(p \mathrm{~A})$ & 6250 & 262 \\
\hline $\mathrm{V}(B I T)(\mathrm{V})$ & 1.0 & 0.686 \\
\hline $\mathrm{V}(\overline{B I T})(\mathrm{V})$ & 0.0 & 0.252 \\
\hline
\end{tabular}




\section{Analysis of Write Operation:}

$\rightarrow$ Transition delay values are as shown in the table

$\rightarrow$ Write operation is not getting affected by the presence of Depletion mode devices

$\rightarrow$ Two reasons,

- Faster $\overline{W L}$ means $\mathrm{V}_{G N D}$ transits to zero even before the access transistors are turned on

- Since bits transit from non-zero initial value to $\mathrm{V}_{H}$, the peak current requirement for the transition is smaller and could be supplied by the single NMOS $_{d e p}$

Transient Analysis Parameters and Response

\begin{tabular}{|c|c|c|c|}
\hline Param & Value & Param & Value \\
\hline \hline$W L t_{r}$ and $t_{f}$ & $100 p s$ & Base $t_{r}$ & $47.0 \mathrm{ps}$ \\
\hline$\overline{W L} t_{r}$ and $t_{f}$ & $10 p s$ & Base $t_{f}$ & $22.0 \mathrm{ps}$ \\
\hline$W L$ Pulse Width & $200 \mathrm{ps}$ & Warm SRAM $t_{r}$ & $50.1 \mathrm{ps}$ \\
\hline$V_{\text {bitpre }}$ & $0.5 \mathrm{~V}$ & Warm SRAM $t_{f}$ & $00.0 \mathrm{ps}$ \\
\hline
\end{tabular}




\section{Analysis of Write Operation (contd.):}

$\rightarrow$ Irrespective of bit state changes, $V_{P W R}$ node and one of the output node $\left(\mathrm{OUT}_{H}\right)$ needs to be pulled up

$\rightarrow$ Considering the capacitance of $\mathrm{V}_{P W R}$ node and OUT $\mathrm{O}_{H}$ node the extra energy would be $327.9^{*} \mathrm{C}_{d i f f}$

$\rightarrow$ For $70 \mathrm{~nm}$ device this would be $36 \mathrm{fJ}$ or $0.14 \mathrm{fJ} / \mathrm{bit}$ which does not change state

$\rightarrow$ Warm SRAM uses more energy when 70 bits or less undergo state transition

$\rightarrow$ This extra energy (36fJ) is insignificant when compared to dynamic energy per access $(0.3 n \mathrm{~J})$, hence we ignored its impact

Write Energy Comparison

\begin{tabular}{|c|c|c|c|c|}
\hline No of Bits & \multicolumn{2}{|c|}{ Energy (fJ) } & \multicolumn{2}{c|}{ Peak Current (mA) } \\
\hline & Base & Warm SRAM & Base & Warm SRAM \\
\hline 256 & 320 & 144 & 5.53 & 0.997 \\
\hline 192 & 240 & 132 & 4.14 & 0.930 \\
\hline 128 & 160 & 118 & 2.75 & 0.840 \\
\hline 64 & 80 & 99 & 1.36 & 0.735 \\
\hline
\end{tabular}




\section{Analysis of Read Operation:}

$\rightarrow$ Tag array access forms the critical path, hence Warm SRAM is used only in Data Array

$\rightarrow$ Since we use Hight- $V_{t}$ access transistors in SRAM cell, access time for precharge voltage of $0.5 \mathrm{~V}$ closely matches with CACTI's estimated value

$\rightarrow$ Bitline delay increases by 4.5X for Warm SRAM, which doesn't increase both cache access time and wave pipelined cycle time

$\rightarrow$ The extra energy estimated in write operation also applies to read

$\rightarrow$ As $V_{P W R}$ node takes finite amount of time to discharge, extra energy depends on the inter-access time 


\section{Analysis of Read Operation (contd.):}

Read Energy w.r.t Inter-Access time

\begin{tabular}{|c|c|c|}
\hline \multicolumn{3}{|c|}{ Base Read Energy: $25.92 f J$} \\
\hline Time $(n s)$ & Energy $(f J)$ & Extra Energy $(f J)$ \\
\hline 25 & 23.99 & -1.93 \\
\hline 50 & 33.86 & 7.94 \\
\hline 75 & 41.56 & 15.64 \\
\hline 100 & 47.22 & 21.30 \\
\hline 125 & 51.38 & 25.46 \\
\hline 150 & 55.27 & 29.35 \\
\hline 175 & 57.45 & 31.53 \\
\hline 200 & 59.44 & 33.52 \\
\hline 300 & 59.44 & 33.52 \\
\hline
\end{tabular}

\section{Discharging of $\mathbf{V}_{P W R}$ node}

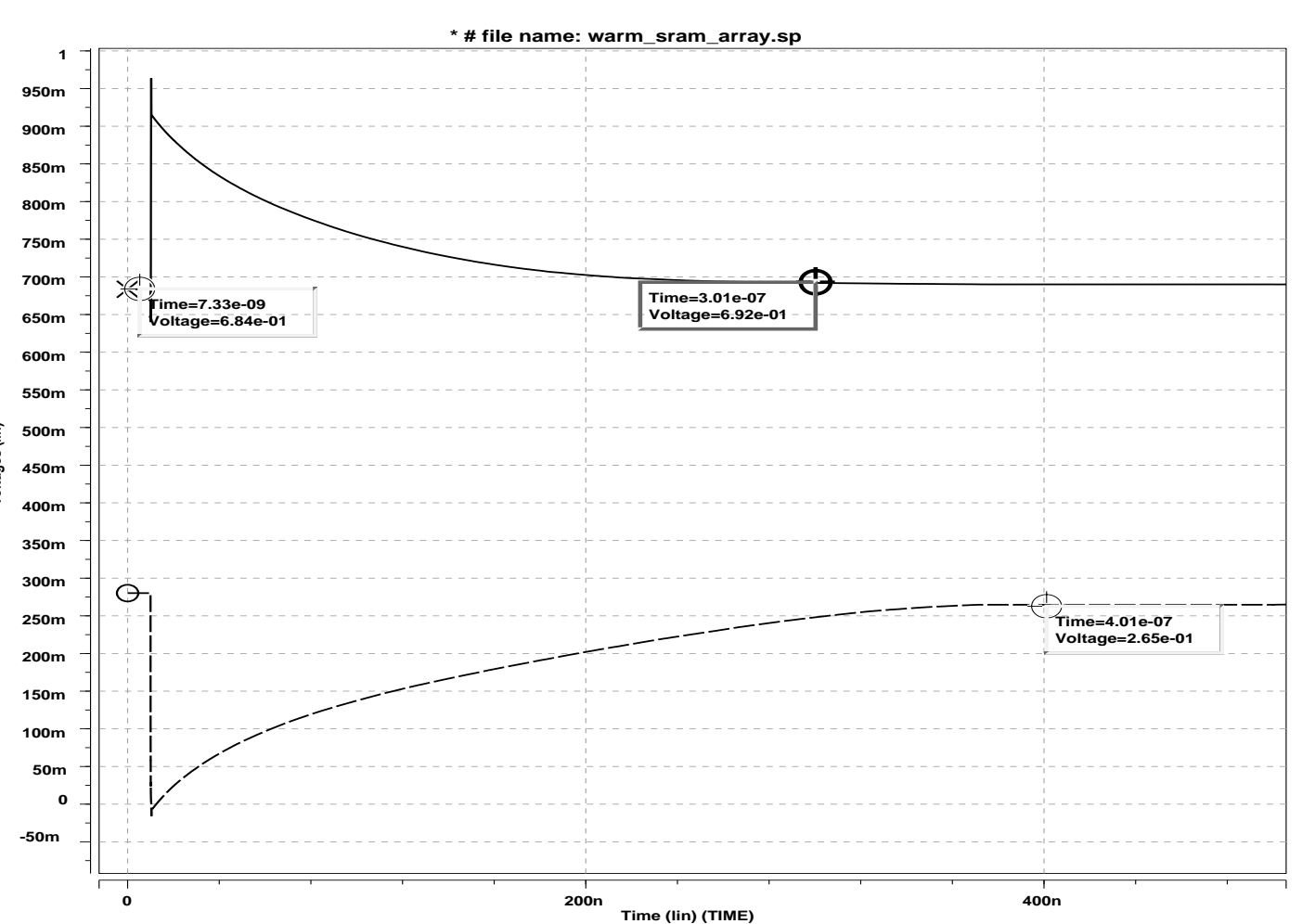


Architecture Level Estimation:

$\rightarrow$ SPEC2000 Integer benchmarks running on Simplescalar 3.0 is used to estimate the energy savings for a hypothetical 32KB,4-way L1 cache

$\rightarrow$ Two sources of extra energy

- Energy to bring Warm SRAM to normal state (max 33.52fJ per access)

- Generation of access control signals ( $\approx 20 \mathrm{fJ}$ per access)

$\rightarrow$ Average net energy savings for $0.5 n s$ cache access time (cycle time) is $94.11 \%$

Access Percentage w.r.t Time

\begin{tabular}{|c|c|c|c|c|c|}
\hline Benchmark & 50 Cycles & 100 Cycles & Benchmark & 50 Cycles & 100 Cycles \\
\hline \hline crafty & 59.73 & 9.15 & eon & 77.91 & 6.06 \\
\hline gcc & 77.85 & 5.47 & twolf & 70.40 & 6.46 \\
\hline gzip & 79.73 & 5.61 & bzip & 86.92 & 4.90 \\
\hline mcf & 68.47 & 11.02 & perlbmk & 77.32 & 3.37 \\
\hline parser & 75.18 & 7.36 & vpr & 69.59 & 7.81 \\
\hline \hline \multicolumn{7}{|c|}{ Avg for 50 Cycles } & & 74.31 & 6.721 \\
\hline \multicolumn{7}{|l}{ Avg for 100 Cycles }
\end{tabular}


Net Energy Savings

\begin{tabular}{|c|c|c|c|c|c|}
\hline Prog & Exec Cycles & Mem Access & Energy Penalty per access $(\mu \mathrm{J})$ & \%Net Saving $(0.2 \mathrm{~ns} / \mathrm{cyc})$ & \%Net Saving $(0.5 \mathrm{~ns} / \mathrm{cyc})$ \\
\hline crafty & 396782412 & 195828079 & 5.93 & 91.28 & 94.02 \\
\hline eon & 350714953 & 240118536 & 6.06 & 90.57 & 93.74 \\
\hline gcc & 393784461 & 223031723 & 5.68 & 91.45 & 94.09 \\
\hline twolf & 444314516 & 172189507 & 4.76 & 92.58 & 94.54 \\
\hline gzip & 277336702 & 169725136 & 4.21 & 91.22 & 94.00 \\
\hline bzip & 269543836 & 185471790 & 4.19 & 91.10 & 93.95 \\
\hline mcf & 487390086 & 195632037 & 5.23 & 92.57 & 94.54 \\
\hline perlbmk & 346674071 & 216796572 & 5.71 & 90.82 & 93.84 \\
\hline parser & 326925643 & 190878110 & 4.91 & 91.26 & 94.01 \\
\hline vpr & 421717636 & 185474202 & 5.09 & 92.16 & 94.37 \\
\hline \hline Avg & 371518431.60 & 197514569.20 & 5.18 & 91.50 & 94.11 \\
\hline
\end{tabular}




\section{MODEL VALIDITY}

$\rightarrow \mathrm{N}_{d}$ (donor concentration) and $\mathrm{d}_{I}$ (implantation depth) could be varied to get the required device characteristics

$\rightarrow$ Two operating points need to be verified

- $\mathrm{NMOS}_{d e p}$ should get cut-off when $\mathrm{V}_{s b}=0.65 \mathrm{~V}$ and $\mathrm{V}_{g}=0 \mathrm{~V}$

- When $\mathrm{V}_{g s}=1 \mathrm{~V}$ the gate should have gain comparable to what is predicted by the enhancement model

$\rightarrow$ The device should operate in Cut-Off or Surface Accumulation region

$\rightarrow$ We solved $\left.\mathrm{V}_{T}\right|_{V_{s b}=0.65}=-0.65 \mathrm{~V}$ for various values of $\mathrm{d}_{I}$ and obtained viable values for $\mathrm{N}_{d}$

$\rightarrow$ For all these values of $\mathrm{N}_{d}$ the requirement $\mathrm{V}_{g_{s}}>\mathrm{V}_{N}$ is met

Process parameters for NMOS $_{d e p}$

\begin{tabular}{|c|c|c|c|c|c|}
\hline$\gamma_{I}$ & $\mathrm{~d}_{I}\left(10^{-10} \mathrm{~m}\right)$ & $\sigma$ & $\mathrm{N}_{d}\left(10^{18} \mathrm{~cm}^{-3}\right)$ & $\mathrm{V}_{T 0}(\mathrm{~V})$ & $\mathrm{V}_{N}(\mathrm{mV})$ \\
\hline \hline $1.5 \gamma$ & 24.21 & 0.625 & 28.2 & -0.6786 & -37.06 \\
\hline $2.0 \gamma$ & 48.41 & 1.5 & 14.23 & -0.6881 & -54.84 \\
\hline $3.0 \gamma$ & 100 & 5 & 5.667 & -0.7084 & -78.78 \\
\hline
\end{tabular}




\section{CONCLUSIONS AND FUtURE WORK}

$\rightarrow$ Static Leakage is one of the biggest challenges facing the semiconductor industry in the near future

$\rightarrow$ We have achieved more than $90 \%$ leakage energy reduction in On-Chip L1 caches without any performance loss

$\rightarrow$ Our technique is immediately applicable to any lower level caches (L2)

$\rightarrow$ On-Chip caches constitute a major fraction of processor's area, hence considerable leakage energy could be saved by using our methodology

$\rightarrow$ Currently investigating the usage of warmup CMOS design style in logic blocks

$\rightarrow$ Working on analytical model capturing the relationship between threshold of depletion devices and leakage reduction 


\section{THANK YOU!!}

\section{Questions?}

